\title{
Taylor-Duffy Method for Singular Tetrahedron-Product Integrals: Efficient Evaluation of Galerkin Integrals for VIE Solvers
}

\author{
M. T. Homer Reid
}

\begin{abstract}
I present an accurate and efficient technique for numerical evaluation of singular 6-dimensional integrals over tetrahedon-product domains, with applications to calculation of Galerkin matrix elements for discretized volume-integralequation (VIE) solvers using Schaubert-Wilton-Glisson (SWG) and other tetrahedral basis functions. My method extends the generalized Taylor-Duffy strategy-used to handle the singular triangle-product integrals arising in discretized surface-integralequation (SIE) formulations-to the tetrahedron-product case; it effects an exact transformation of a singular 6-dimensional integral to an nonsingular lower-dimensional integral that may be evaluated by simple numerical cubature The method is highly general and may-with the aid of automatic code generation facilitated by computer-algebra systems-be applied to a wide variety of singular integrals arising in various VIE formulations with various types of tetrahedral basis function, of which I present several examples. To demonstrate the accuracy and efficiency of my method, I apply it to the calculation of matrix elements for the volume electric-field integral equation (VEFIE) discretized with SWG basis functions, where the method yields 12-digit or higher accuracy with low computational cost-an improvement of many orders of magnitude compared to existing techniques.
\end{abstract}

\section{INTRODUCTION}

In this paper I present an efficient technique for evaluating singular 6-dimensional integrals over tetrahedron-product domains, such as those commonly encountered in discretized volume-integral equation (VIE) formulations [1]-[3] with tetrahedral basis functions [4]-[6]. My method extends the generalized Taylor-Duffy method for singular triangle-product integrals $[7]-[9]$ to the tetrahedron-product case, exactly transforming singular 6-dimensional integrals to nonsingular lowerdimensional integrals amenable to simple numerical cubature. I formulate the basic algorithm, show how it may be applied to several distinct VIE formulations, and present computational results demonstrating its accuracy and efficiency; for the specific case of singular VEFIE integrals [2] with SWG basis functions [6] (defined below) I obtain 12 or more digits of accuracy with modest computational cost, an improvement of many orders of magnitude compared to a recently-proposed alternative approach [10].

Discretized VIE methods [1]-[3] using tetrahedral basis functions [5], [6] are useful for attacking many problems in science and engineering, including electromagnetic scattering [11], [12], acoustic wave propagation [4], inductance

M. T. Homer Reid is with the Department of Mathematics, Massachusetts Institute of Technology. extraction [13], and fluctuation-induced phenomena [14]. Although there exists a considerable variety of VIE formulations [4], [15]-[17] and multiple choices of tetrahedral basis functions-including piecewise-constant [4], piecewiselinear [5], and SWG functions [6]- a computational challenge common to all Galerkin VIE formulations is the need for accurate and efficient numerical evaluation of 6-dimensional tetrahedron-product integrals, typically of the general form

$$
\mathcal{I}=\int_{\mathcal{T}} d \mathbf{x} \int_{\mathcal{T}^{\prime}} d \mathbf{x}^{\prime} P\left(\mathbf{x}, \mathbf{x}^{\prime}\right) K\left(\left|\mathbf{x}-\mathbf{x}^{\prime}\right|\right)
$$

where $\mathcal{T}, \mathcal{T}^{\prime}$ are tetrahedra, $P$ is a polynomial in the cartesian components of $\mathbf{x}, \mathbf{x}^{\prime}$, and $K(r)$ is a scalar kernel function. In the commonly-encountered case in which $\mathcal{T}, \mathcal{T}^{\prime}$ have one or more common vertices and $K(r)$ is singular at $r=0$, the integral (1) cannot be evaluated by simple numerical cubature [18]; instead, more sophisticated integration strategies are required, whose accuracy and efficiency play a large part in determining those of the overall VIE solver [15].

To date, several methods for evaluating singular tetrahedronproduct integrals have been discussed; many such methods, including that proposed here, extend techniques originally developed for singular triangle-product integrals [like (1) but with $\mathcal{T}, \mathcal{T}^{\prime}$ replaced by two-dimensional triangular domains], a problem that has been studied for decades due to its importance for surface-integral-equation (SIE) solvers [2], [19], [20]. Strategies proposed for singular tetrahedon-product integrals include singularity subtraction [21], [22], separation of inner and outer 3D integrals with the former (latter) evaluated analytically (numerically) [23], and the use of Stokes' theorem [24] to recast volume integrals as surface integrals [10].

The method of Bleszynski et al. [10] is particularly attractive in that it effects an exact transformation of the singular 6-dimensional integral (1) to a sum of non-singular 4dimensional integrals amenable to straightforward low-order numerical cubature. However, the method was presented in Ref. 10 only for one particular VIE formulation with particular basis functions \{namely, the volume electric-field integral equation (VEFIE) [16], [17] with SWG functions [6]\}, and it is unclear if or how the method could be used to evaluate the integrals arising in formulations. Moreove, the number of 4-dimensional integrals that must be evaluated is large (as many as 16), and these integrals - though nonsingularconverge relatively slowly in numerical quadrature schemes, a point that was noted already in Ref. 10 and which I corroborate 
and discuss in further detail below (see Section III-B and Figure 11.

In this paper I propose a strategy that, like that of Ref. 10 . exactly transforms (1) into a sum of non-singular lowerdimensional integrals, which are evaluated by low-order numerical cubature in a practical solver. However, in contrast to the Stokes'-theorem underpinning of Ref. 10, my method is based on Duffy's singularity-cancellation technique [7], which was applied to triangle-product integrals originally by Taylor [8] and later in more generality by Ref. 9; here (Section II) I extend these ideas to the tetrahedron-product case, culminating in a nonsingular reduced-dimensional integral [equation (9p] that is exactly equivalent to (11). This reduction scheme offers several advantages (Section III): (a) I formulate the algorithm in full generality for any integral of the form (1), offering immediate application to many VIE formulations and choices of basis function; some explicit examples are given in Section III-A. (b) The dimension of the reduced integral produced by this method is $D=6-N_{\mathrm{Cv}}$ in general and $D=5-N_{\mathrm{CV}}$ for power-law kernels $\left[K(r) \sim r^{p}\right]$, where $N_{\mathrm{CV}}=\{1,2,3,4\}$ is the number of vertices common to $\mathcal{T}, \mathcal{T}^{\prime}$; in the commontetrahedron $\left(N_{\mathrm{Cv}}=4\right)$ and common-face $\left(N_{\mathrm{Cv}}=3\right)$ cases this results in final integrals of dimension $D=2$ or $D=3(D=1$ or 2 for power-law kernels), a further reduction of dimension than is achieved by the method of Ref. 10. (c) The number of $D$-dimensional integrals into which (1) is transformed is at most 18 and as few as 9 in some cases; moreover, all integrals extend over the same region of integration-namely, the unit $D$-dimensional hypercube - and may thus be combined into a single integral, affording significant efficiency through reuse of computation. Thus my method is not only quite general but also highly efficient, as I demonstrate with illustrative computational results in Section III-B, Questions for future work are discussed in Section IV] and technical details are relegated to Appendices.

The algorithm of this paper is implemented in BUFF-EM a free, open-source software implementation of the VEFIE with SWG basis functions [25].

\section{EXTENSION OF TAYLOR-DUfFy METHOD to TETRAHEDRON-PRODUCT INTEGRALS}

The method of Duffy transforms [7] was applied to the desingularization and dimensional reduction of singular triangle-product integrals by Taylor [8] and later in more generality by Ref. 9 . In this section I show that the same basic ideas may be used to desingularize and reduce the dimension of tetrahedron-product integrals; the result is equation (9), a nonsingular reduced-dimensional integral that is exactly equivalent to the singular six-dimensional integral (1).

The logical flow of the transformation procedure is identical to that of Refs. 8,9 and proceeds as follows. (a) Subdivide the tetrahedron-product domain in (1) into $D$ subdomains and change integration variables to ensure that each subdomain is a product of tetrahedra with one vertex at the origin, facilitating Duffy transformation [Section $[\overline{I I-A}]$ ]. (For the tetrahedronproduct case we have $D=18$, in contrast to $D=6$ for the triangle-product case [8], [9].) (b) Within each subdomain, analytically evaluate the integrals over all variables of which the kernel $K(r)$ is independent [Section [II-B]]. (As in the triangle-product case, there are $N_{\mathrm{cv}}-1$ such variables, where $N_{\mathrm{cv}}$ is the number of vertices common to $\mathcal{T}, \mathcal{T}^{\prime}$.) (c) Within each subdomain, perform a Duffy transformation, analytically evaluate the integral over the untransformed variable, then combine the remaining integrals for each subdomain into a single integral over the $\left(6-N_{\mathrm{CV}}\right)$-dimensional unit hypercube [Section (III-C)] to yield the final master formula (9).

As in the triangle-product case, the reduction procedure is straightforward but tedious and error-prone if carried out by hand, in practice requiring the use of automatic code generation facilitated by computer algebra systems (Section II-D).

Although the TD reduction method may be used for all tetrahedron pairs with $N_{\mathrm{CV}}=1$ or more common vertices, here I formulate it only for the case of $N_{\mathrm{CV}} \geq 2$, as the significant cost of implementing the TD method seem not to be justified by the modest reduction in computational cost it affords in the common-vertex case.

\section{A. Decomposition into tetrahedron-product subdomains}

The goal of this step is to decompose the tetrahedronproduct domain in (1) as the union of $D$ tetrahedron-product subdomains,

$$
\mathcal{T} \times \mathcal{T}^{\prime}=\bigcup_{d=1}^{D} \triangle_{d} \times \bar{\triangle}_{d},
$$

with the property that $\triangle_{d}$ and $\bar{\triangle}_{d}$ each have one vertex at the origin of coordinates, as required to allow Duffy transformation.

In (1) first make the change of variables $\left(\mathbf{x}, \mathbf{x}^{\prime}\right) \rightarrow(\boldsymbol{\xi}, \boldsymbol{\eta})$ where $\boldsymbol{\xi}, \boldsymbol{\eta}$ run over a standard tetrahedron $\triangle_{0}$ :

$$
\mathcal{I}=J \int_{\triangle_{0}} d \boldsymbol{\xi} \int_{\triangle_{0}} d \boldsymbol{\eta} P\left(\mathbf{x}(\boldsymbol{\xi}), \mathbf{x}^{\prime}(\boldsymbol{\eta})\right) K(r(\boldsymbol{\xi}, \boldsymbol{\eta}))
$$

where

$$
\int_{\triangle_{0}} d \boldsymbol{\xi}=\int_{0}^{1} d \xi_{1} \int_{0}^{\xi_{1}} d \xi_{2} \int_{0}^{\xi_{2}} d \xi_{3}
$$

and

$$
\begin{aligned}
\mathbf{x}(\boldsymbol{\xi}) & =V_{1}+\xi_{1} \mathbf{L}_{1}+\xi_{2} \mathbf{L}_{2}+\xi_{3} \mathbf{L}_{3} \\
\mathbf{x}^{\prime}(\boldsymbol{\eta}) & =V_{1}+\eta_{1} \mathbf{L}_{1}^{\prime}+\eta_{2} \mathbf{L}_{2}^{\prime}+\eta_{3} \mathbf{L}_{3}^{\prime} \\
r(\boldsymbol{\xi}, \boldsymbol{\eta}) & =\left|\mathbf{x}(\boldsymbol{\xi})-\mathbf{x}^{\prime}(\boldsymbol{\eta})\right|
\end{aligned}
$$

with

$\begin{array}{lll}\mathbf{L}_{1}=\left(\mathbf{V}_{2}-\mathbf{V}_{1}\right), & \mathbf{L}_{2}=\left(\mathbf{V}_{3}-\mathbf{V}_{2}\right), & \mathbf{L}_{3}=\left(\mathbf{V}_{4}-\mathbf{V}_{3}\right) \\ \mathbf{L}_{1}^{\prime}=\left(\mathbf{V}_{2}^{\prime}-\mathbf{V}_{1}\right), & \mathbf{L}_{2}^{\prime}=\left(\mathbf{V}_{3}^{\prime}-\mathbf{V}_{2}^{\prime}\right), & \mathbf{L}_{3}^{\prime}=\left(\mathbf{V}_{4}^{\prime}-\mathbf{V}_{3}^{\prime}\right)\end{array}$ and $J=36 \mathcal{V} \mathcal{V}^{\prime}$. Here $\left\{\mathbf{V}_{i}, \mathbf{V}_{i}^{\prime}\right\}$ and $\mathcal{V}, \mathcal{V}^{\prime}$ are the vertices and volumes of $\triangle, \triangle^{\prime}$. We have assumed that $\mathbb{T}, \mathbb{T}^{\prime}$ have at least one common vertex, labeled $\mathbf{V}_{1}$, and the remaining vertices should be ordered such that common vertices have lower indices than non-common vertices; thus for the common-edge case we have $\mathbf{V}_{2}^{\prime}=\mathbf{V}_{2}$, for the common-triangle case we have additionally $\mathbf{V}_{3}^{\prime}=\mathbf{V}_{3}$, and for the common-tetrahedron case we have additionally $\mathbf{V}_{4}^{\prime}=\mathbf{V}_{4}$. Note that $r(\boldsymbol{\xi}, \boldsymbol{\eta})$ is the 
square root of a homogeneous second-degree polynomial in the components of $\boldsymbol{\xi}, \boldsymbol{\eta}$ :

$$
r(\boldsymbol{\xi}, \boldsymbol{\eta})=\sqrt{R_{i j}^{\xi \xi} \xi_{i} \xi_{j}+R_{i j}^{\xi \eta} \xi_{i} \eta_{j}+R_{i j}^{\eta \eta} \eta_{i} \eta_{j}}
$$

where the $\left\{R_{i j}\right\}$ coefficients are functions of the geometric parameters; for example, $R_{12}^{\xi \xi}=2 \mathbf{L}_{1} \cdot \mathbf{L}_{2}$. The homogeneity of the polynomial under the radical, which follows from the fact that $\mathbf{T}, \mathbf{T}^{\prime}$ have one or more common vertices, is what allows analytical evaluation of the $w$ integral below (Section II-C).

Following Ref. 8, now introduce the relative coordinates $\mathbf{u} \equiv \boldsymbol{\eta}-\boldsymbol{\xi}$, change variables from $\{\boldsymbol{\xi}, \boldsymbol{\eta}\}$ to $\{\boldsymbol{\xi}, \mathbf{u}\}$, and decompose the domain of integration into $D$ subdomains with the property that, within each subdomain, both $\mathbf{u}$ and $\boldsymbol{\xi}$ run over tetrahedra with one vertex at the origin:

$\mathcal{I}=\sum_{d=1}^{D} \int_{\triangle_{d}^{\mathbf{u}}} d \mathbf{u} \int_{\triangle_{d}^{\boldsymbol{\xi}}} d \boldsymbol{\xi} P\left(\mathbf{x}(\boldsymbol{\xi}), \mathbf{x}^{\prime}(\boldsymbol{\xi}+\mathbf{u})\right) K(r(\boldsymbol{\xi}, \boldsymbol{\xi}+\mathbf{u}))$

The corresponding step in the triangle-product case [8], [9] similarly writes the original triangle-product integral as a sum of integrals over triangle-product subdomains, with both triangles in each subdomain having one vertex at the origin [8]. However, whereas that case involves $D=6$ triangle-product subdomains, for the tetrahedron-product case one finds that the minimum number of subdomains allowing (1) to be decomposed in the form (4) is $D=18$. (For the commontetrahedron case this number may be reduced to $D=9$ by identifying pairs of identical subdomains; this is analogous to the reduction from $D=6$ to $D=3$ available for the $N_{\text {CV }}=3$ case of the Taylor-Duffy approach to triangle-product integrals [8]).

Explicit definitions of the 18 tetrahedral subdomains $\triangle_{d}^{\mathbf{u}}, \triangle_{d}^{\boldsymbol{\xi}}$ are given in the Appendix (Tables III IV.

\section{B. Analytical evaluation of $\boldsymbol{\xi}$ integrals}

If the original tetrahedra have 2 or more common vertices, the distance function $r$ in 2 is independent of one or more of the $\boldsymbol{\xi}$ variables. [For example, in the common-edge case $\left(N_{\mathrm{CV}}=2\right) r$ is independent of $\xi_{1}$, while in the commontetrahedron case $\left(N_{\mathrm{CV}}=4\right) r$ is independent of all $\boldsymbol{\xi}$ variables.] The kernel factor $K(r)$ in (4) may then be pulled out of the integrals over those variables, leaving integrals over just the polynomial $P$; these may be evaluated analytically to yield new polynomials $\bar{P}$ depending on just the remaining variables:

$$
\begin{aligned}
\bar{P}_{d}^{4 \mathrm{CV}}\left(\mathbf{u}_{d}\right) & \equiv \int d \xi_{3} \int d \xi_{2} \int d \xi_{1} P\left(\boldsymbol{\xi}, \mathbf{u}_{d}+\boldsymbol{\xi}\right) \\
\bar{P}_{d}^{3 \mathrm{CV}}\left(\mathbf{u}_{d}, \xi_{3}\right) & \equiv \int d \xi_{2} \int d \xi_{1} P\left(\boldsymbol{\xi}, \mathbf{u}_{d}+\boldsymbol{\xi}\right) \\
\bar{P}_{d}^{2 \mathrm{CV}}\left(\mathbf{u}_{d}, \xi_{2}, \xi_{3}\right) & \equiv \int d \xi_{1} P\left(\boldsymbol{\xi}, \mathbf{u}_{d}+\boldsymbol{\xi}\right) .
\end{aligned}
$$

This reduces the dimension of the integral by $N_{\mathrm{CV}}-1$.

\section{Duffy Transformation and evaluation of $w$ integral}

For each of the $D$ subregions I now make a Duffy transformation [7]-that is, for $1 \leq d \leq D$ I introduce functions

$$
\mathbf{u}_{d}=\mathbf{u}_{d}(w, \mathbf{y}), \quad \boldsymbol{\xi}_{d}=\boldsymbol{\xi}_{d}(w, \mathbf{y})
$$

and make the change of variables $\left(\mathbf{u}_{d}, \boldsymbol{\xi}_{d}\right) \rightarrow(w, \mathbf{y})$ in the $d$ th subregion integral; here the dimension of the $\mathbf{y}$ vector is $Y \equiv 6-N_{\mathrm{CV}}$. The Duffy transformations for each subregion are tabulated in the Appendix (Tables V.VII).

As in the triangle-product case [8], [9], the key property of this transformation is that, when expressed as functions of the new variables, each component of $\mathbf{u}$ and $\boldsymbol{\xi}$ is proportional to $w$. This yields a Jacobian factor for the $d$ th subdomain of the form $J_{d}(w, \mathbf{y}) \equiv w^{Y} \mathcal{J}_{d}(\mathbf{y})$ and-in view of the homogeneity of the polynomial in $(3)$-allows the quantity $w$ to be extracted from the square root in equation (3):

$$
r(\boldsymbol{\xi}, \mathbf{u})=w X_{d}(\mathbf{y})
$$

with $X_{d}(\mathbf{y})$ nonvanishing over the region of integration. Also, the $\bar{P}$ polynomials defined by 5 may be expanded as power series in $w$, with $\mathbf{y}$-dependent coefficients:

$$
\bar{P}_{d}(\mathbf{u}, \boldsymbol{\xi}) \equiv \sum_{n} \mathcal{P}_{d n}(\mathbf{y}) w^{n}
$$

Finally, because the domain of integration for the Duffytransform variables $(w, \mathbf{y})$ is the same for all $D$ subdomains (namely, $0 \leq w, y_{i} \leq 1$ ), the order of summation and integration in (2) may be reversed to yield a single integral whose integrand is a sum of $D$ terms; using (7) and (8), the final transformed version of the original integral (1) then reads

$$
\mathcal{I}=\int_{\square Y} \sum_{d=1}^{D} \mathcal{J}_{d}(\mathbf{y}) \sum_{n} \mathcal{P}_{d n}(\mathbf{y}) \mathcal{K}_{n+Y}\left(X_{d}(\mathbf{y})\right) d \mathbf{y}
$$

where the $\{\mathcal{K}\}$ functions are the "first integrals" of $K$, defined by [9]

$$
\mathcal{K}_{p}(X) \equiv \int_{0}^{1} w^{p} K(w X) d w
$$

If the kernel $K(r)$ has a singularity of degree $q$ at the origin [i.e. $K(r) \sim \frac{1}{r^{q}}$ as $r \rightarrow 0$ ] then $\mathcal{K}_{p}$ exists and is nonsingular for $p \geq q$; equation (9) thus desingularizes all integrals of the form $\sqrt{1}$ with singularities as strong as $\left\{\frac{1}{r^{2}}, \frac{1}{r^{3}}, \frac{1}{r^{4}}\right.$ for the common- $\{$ tetrahedron, face, edge $\}$ cases. If the polynomial $P\left(\mathbf{x}, \mathbf{x}^{\prime}\right)$ vanishes at $\mathbf{x}=\mathbf{x}^{\prime}$, then the sum over $n$ in 90 begins at $n=1$ or higher, in which case kernels with even stronger singularities are desingularized by (9); an example is given in Section III-A.

As noted in Refs. 8, 9, the first integral (10) may be evaluated in closed form for many kernels relevant to VIE solvers, including the Helmholtz kernel $\frac{e^{i k r}}{4 \pi r}$ and its gradient; explicit expressions for $\mathcal{K}_{n}$ for various kernels of interest may be found in [9]. For some kernels - in particular, power-law kernels of the form $K(r) \sim r^{p}$ for integer $p$-the dimension of the transformed integral (9) may be further reduced by evaluating one of the $\mathbf{y}$ integrals analytically, yielding a final reduced integral of dimension $\{1,2,3\}$ for the common- $\{$ tetrahedron, face, edge $\}$ cases. This is useful for applications to singularitysubtraction methods [22] or frequency-caching schemes [9] in 
which the contributions of individual terms in the power-series expansion of $K(r)$ around $r=0$ are computed analytically, yielding integrals of the form (1) with $K(r) \sim r^{p}$ for various powers $p$. The procedure for effecting this further reduction of (9) is identical to that presented in Ref. 9 for the triangleproduct case.

On the other hand, even for cases in which the kernel is so complicated that even the first integrals (10) cannot be evaluated in closed form, equation $(9)$ together with $(10)$ may still constitute a useful exact transformation of the original integral (1); if the original integral has integrable singularities that prevent direct application of numerical cubature, equations (9) and (10) define a non-singular integral to which straightforward numerical cubature may be applied directly.

\section{Automation by computer algebra system}

The reduction procedure outlined above, though conceptually straightforward, in practice requires large numbers of elementary calculus and algebra manipulations that are tedious and error-prone if carried out by hand. Indeed, to obtain the integrand of the reduced integral $(9)$ for a given polynomial $P$ and kernel $K$ we must-for each of the $D$ subregions-(a) evaluate the integrals in (5) to compute the functions $\bar{P}_{d}(\mathbf{u}, \boldsymbol{\xi})$, (b) use the $d$-dependent Duffy transformation (6) to rewrite in terms of $(w, \mathbf{y})$, (c) series-expand in $w$ to identify the coefficient functions $\mathcal{P}_{d n}(\mathbf{y})$ in $(8)$, then (d) pair each $\mathcal{P}$ with the appropriate $\mathcal{J}$ and $\mathcal{K}$ factors and sum over subdomains $d$ to construct a function of $\mathbf{y}$ that may be passed to a numerical cubature routine as the integrand of (9) The resulting integrand routinely consists of hundreds of terms, with the complexity increasing with that of the polynomial $P$ in (1); attempts to construct this function by hand are clearly hopeless.

As in the triangle-product case [9], the solution is to make avail to code generation by computer algebra systems such as MATHEMATICA or MAXIMA, which are ideally suited to carrying out steps (a-d) above automatically and emitting code defining the integrand of (9). This approach was used to implement the method of this paper in the BUFF-EM FVC solver [25], and the online documentation for that solver includes sample MATHEMATICA codes implementing the codegeneration process for particular cases of (1).

\section{Applications to GALERKIN VIE Formulations WITH TETRAhEDRAL BASIS FUnCTIONS}

The Taylor-Duffy method proposed in this paper is directly applicable to the computation of matrix elements for a variety of Galerkin-discretized VIE formulations using tetrahedral basis functions. Here I first give the specific forms of the $P$ polynomial and $K$ kernel in (1) for several popular VIE formulations (Section III-A), then present computational results for the particular case of the VEFIE formulation [26] with SWG basis functions [6] (Section [II-B). As I show, the reduced integral (9) produced by the Taylor-Duffy transformation in this case may be evaluated to high (12-digit or greater) accuracy with only $\sim 20$ quadrature points per dimension, an improvement of many orders of magnitude compared to another recently-proposed technique for singular tetrahedronproduct integrals [10].

\section{A. $P$ and $K$ functions for various VIE formulations}

AIM acoustic-wave solver with tetrahedron-pulse functions. For the adaptive integral method (AIM) acoustic-wave VIE formulation with piecewise-constant (pulse) tetrahedral basis functions [4], elements of the stiffness matrix take the form of (1) with the $P$ and $K$ functions given by

$$
P^{\mathrm{AIM}}\left(\mathbf{x}, \mathbf{x}^{\prime}\right)=1, \quad K^{\mathrm{AIM}}(r)=\frac{e^{i k r}}{4 \pi r}
$$

with $k$ the acoustic wavenumber in the background medium.

VEFIE with SWG functions. For the volume electric-field integral equation (VEFIE) [26] discretized with SWG basis functions [6], each element of the system matrix is a sum of four tetrahedon-product integrals of the form (1) with the $P$ and $K$ functions given by

$$
\begin{aligned}
P^{\mathrm{EFIE}}\left(\mathbf{x}, \mathbf{x}^{\prime}\right) & =(\mathbf{x}-\mathbf{Q}) \cdot\left(\mathbf{x}^{\prime}-\mathbf{Q}^{\prime}\right)-\frac{9}{k^{2}}, \\
K^{\mathrm{EFIE}}(r) & =\frac{e^{i k r}}{4 \pi r}
\end{aligned}
$$

Here $\mathbf{Q}, \mathbf{Q}^{\prime}$ are the source/sink vertices of the $\mathrm{SWG}$ functions and $k$ is the vacuum photon wavenumber.

VMFIE with SWG functions. For the volume magnetic-field integral equation (VMFIE) [27] with SWG basis functions, each element of the system matrix is a sum of four tetrahedonproduct integrals of the form (1) with

$$
\begin{aligned}
P^{\mathrm{MFIE}}\left(\mathbf{x}, \mathbf{x}^{\prime}\right) & =(\mathbf{x}-\mathbf{Q}) \cdot\left[\left(\mathbf{x}-\mathbf{x}^{\prime}\right) \times\left(\mathbf{x}^{\prime}-\mathbf{Q}^{\prime}\right)\right] \\
K^{\mathrm{MFIE}}(r) & =(i k r-1) \frac{e^{i k r}}{4 \pi r^{3}} .
\end{aligned}
$$

The $\frac{1}{r^{3}}$ singularity of $K^{\mathrm{MFIE}}(r)$ at the origin might appear to preclude application of the TD reduction method for this kernel in the common-tetrahedron case (for which, as noted in the previous section, we are guaranteed only desingularization of kernels with singularities of $\frac{1}{r^{2}}$ or weaker). However, the vanishing of $P^{\mathrm{MFIE}}$ at $\mathbf{x}=\mathbf{x}^{\prime}$ affords extra leeway (by ensuring that the sum over $n$ in (9) begins at $n=1$ ), allowing the pairing $\left\{P^{\mathrm{MEFIE}}, K^{\mathrm{MEFIE}}\right\}$ to be desingularized with no difficulty in the common-tetrahedron and all other cases.

\section{B. Computation of VEFIE-SWG matrix elements: Comparison to Method of Bleszynski et al.}

As a concrete demonstration of the accuracy and efficiency of the Taylor-Duffy (TD) method proposed here, I now use it to compute the contributions of specific tetrahedron pairs to VEFIE matrix elements between SWG basis functionsthat is, for fixed tetrahedra $\mathcal{T}, \mathcal{T}^{\prime}$ I evaluate equation (1) with the $P$ and $K$ functions of equation (11) at wavenumber $k=10$, corresponding to a wavelength on the order of one-half the tetrahedron edge length. For comparison, I also evaluate the same integrals using my own implementation of the recently proposed surface-integral-reduction (SIR) method of Bleszynski et al. [10].

I consider three pairs of tetrahedra with $N_{\mathrm{CV}}=\{4,3,2\}$ common vertices (the common- $\{$ tetrahedron, face, edge $\}$ cases); Table I] lists the vertices of these tetrahedra. (Note that I have chosen tetrahedron $\mathcal{T}_{\mathrm{A}}$ to be the tetrahedron used 


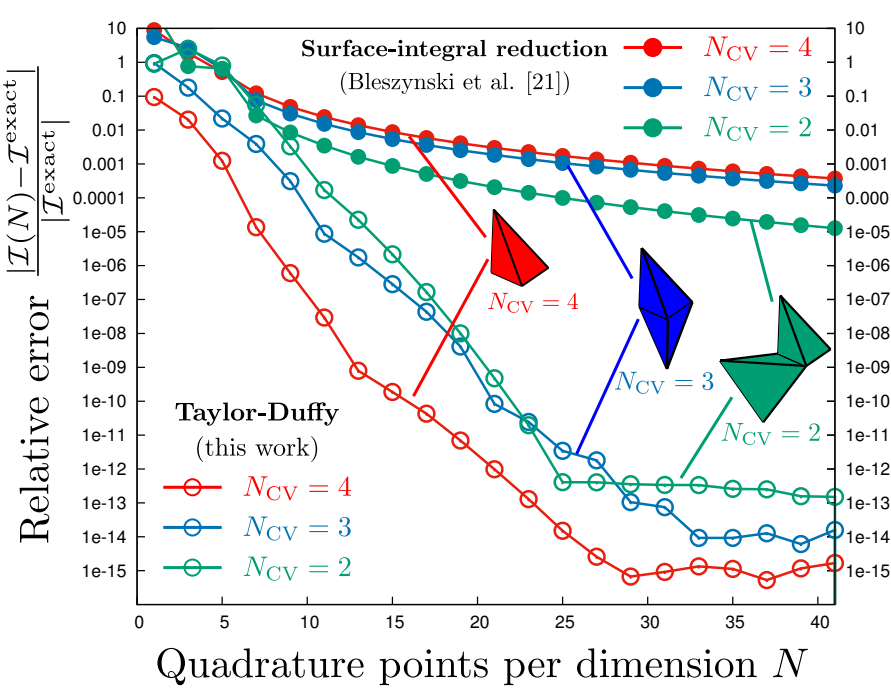

Fig. 1. Comparison of convergence rates for the Taylor-Duffy (TD) method proposed in this paper and for the surface-integral-reduction (SIR) scheme of Bleszynski et al. [10] as used to evaluate VEFIE matrix elements [equation (1) with the $P$ and $K$ functions of equation [11] at wavenumber $k=10$ in units where the tetrahedron edge lengths are of order $\sim 1$. For pairs of tetrahedra with $N_{\mathrm{CV}}=\{4,3,2\}$ common vertices (inset; see also Table If, I use the TD and SIR methods to transform the singular 6-dimensional integral (1) into a nonsingular $M$-dimensional integral (where $M=4$ for the SIR method and $M=6-N_{\mathrm{CV}}=\{2,3,4\}$ for the TD method), then evaluate this integral numerically using nested Clenshaw-Curtis quadrature with $N$ points per dimension (for a total of $N^{M}$ integrand samples) to obtain an approximation $\mathcal{I}(N)$ to the original integral (1). Plotted is the relative error in this approximation vs. $N$. The TD method converges exponentially with $N$, yielding 12 or more digits of accuracy for $N \approx 25$, and is several orders of magnitude more accurate than the SIR method for all $N>10$. For example, in the $N_{\mathrm{CV}}=4$ case with $N=15$ the SIR method requires $15^{4}=50,625$ cubature points to achieve 2-digit accuracy, while the TD method requires $15^{2}=225$ points to achieve 10-digit accuracy. (As discussed in the text, the computational cost per integrand sample is comparable for the two methods.)

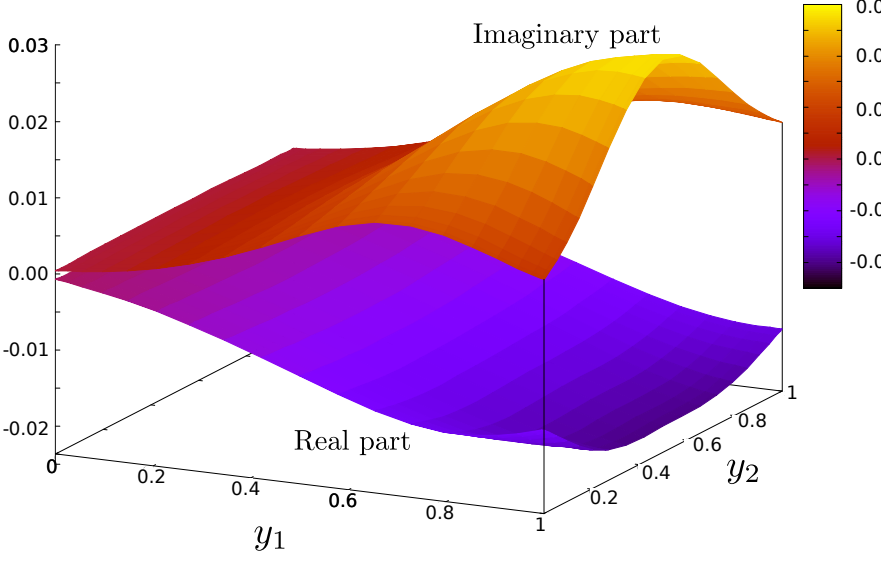

Fig. 2. Integrand of the reduced integral 9 for the common-tetrahedron $\left(N_{\mathrm{CV}}=4\right)$ case of Figure 1 Whereas the integrand of the original 6dimensional integral (1) has both integrable singularities and sinusoidal variations at a wavelength $(\lambda=2 \pi / 10)$ shorter than the linear size $(L \sim 1)$ of the tetrahedron, the Taylor-Duffy reduction process achieves a tremendous amount of smoothing; the gentle variation of the reduced integrand with $\mathbf{y}$ explains why only low-order cubature is required to evaluate the integral to 15-digit accuracy (Figure 1 .

\begin{tabular}{|c|c|c|c|}
\hline & $\mathcal{T}_{A}$ & $\mathcal{T}_{B}$ & $\mathcal{T}_{C}$ \\
\hline $\mathbf{V}_{2}$ & $(1,0,0)$ & $(1,0,0)$ & $(0,0,1)$ \\
\hline $\mathbf{V}_{3}$ & $(0,1,0)$ & $(0,1,0)$ & $(-0.04,-1.09,-0.05)$ \\
\hline $\mathbf{V}_{4}$ & $(0,0,1)$ & $(0.3,0.4,-1.03)$ & $(0.3,-0.4,-1.09)$ \\
\hline
\end{tabular}

TABLE I

VERTiCES OF TETRAHEdRa USED FOR SAMPle CALCUlations. All TETRAHEDRA HAVE ONE VERTEX AT THE ORIGIN, $\mathbf{V}_{1}=(0,0,0)$. THE

COMMON- $\{$ TETRAHEDRON, FACE, EDGE $\}$ CASES $\left(N_{\mathrm{CV}}=\{4,3,2\}\right)$ CORRESPOND TO TETRAHEDRON PAIRS $\left\{\left(\mathcal{T}_{A}, \mathcal{T}_{A}\right),\left(\mathcal{T}_{A}, \mathcal{T}_{B}\right),\left(\mathcal{T}_{A}, \mathcal{T}_{C}\right)\right\}$.

\begin{tabular}{c|c|c|c}
$\left(\mathcal{T}, \mathcal{T}^{\prime}\right)$ & $N_{\mathrm{CV}}$ & $\operatorname{Re} \mathcal{I}^{\text {exact }}$ & $\operatorname{Im} \mathcal{I}^{\text {exact }}$ \\
\hline$\left(\mathcal{T}_{A}, \mathcal{T}_{A}\right)$ & 4 & $-7.8624620487335 \mathrm{e}-04$ & $+8.5795441769385 \mathrm{e}-04$ \\
$\left(\mathcal{T}_{A}, \mathcal{T}_{B}\right)$ & 3 & $+4.2568610165422 \mathrm{e}-05$ & $+3.2199164645680 \mathrm{e}-05$ \\
$\left(\mathcal{T}_{A}, \mathcal{T}_{C}\right)$ & 2 & $-3.0105189689052 \mathrm{e}-05$ & $-7.1022045556570 \mathrm{e}-07$
\end{tabular}

TABLE II

REFERENCE VALUES OF INTEgRAL 1 WITH THE $P$ AND $K$ FUNCTIONS OF EQUATIONS 11 ( $k=10)$, OBTAINED BY NESTED CLENSHAW-CURTIS QUADRATURE OF THE REDUCED TAYLOR-DUFFY INTEGRAL 9 WITH $N=51$ QUADRATURE POINTS PER DIMENSION.

by [10] in SIR studies of the $N_{\mathrm{CV}}=4$ case, allowing direct comparison with results reported there.) The TD and SIR methods reduce the 6-dimensional singular integral (11) to a nonsingular $M$-dimensional integral (where $M=4$ for the SIR method and $M=6-N_{\mathrm{CV}}=\{2,3,4\}$ for the TD method), which I evaluate numerically using nested Clenshaw-Curtis (CC) quadrature [28] with $N$ points per dimension (total of $N^{M}$ integrand samples) to obtain an approximation $\mathcal{I}(N)$ to the original integral (1). (The SIR method involves integrals over triangles, which I reparameterize as integrals over the unit square to allow nested $\mathrm{CC}$ quadrature.)

Figure 1 plots the relative error $\mathcal{E}(N) \equiv \mid \mathcal{I}(N)-$ $\mathcal{I}^{\text {exact }}|/| I^{\text {exact }} \mid$ versus $N$ for the TD and SIR methods. (Ref.03 erence values $\mathcal{I}^{\text {exact }}$ are the results of TD calculations with $02 N=51$ and are tabulated in Table II]) My results for the SIR method in the $N_{\mathrm{CV}}=4$ case agree with the results of Ref. 10 . which reported relative errors of $10^{-2}$ for $N=12$ and $10^{-3}$ for $N=40$. As the total number of cubature points used by the SIR method in these cases are $N_{\text {tot }}=12^{4} \approx 2 \cdot 10^{4}$ and $N_{\text {tot }}=40^{4} \approx 3 \cdot 10^{6}$, the tenfold reduction in error requires more than a hundredfold increase in cost; the SIR method for this case appears to be converging at the extremely slow algebraic rate $\mathcal{E} \sim 1 / \sqrt{N_{\text {tot }}}$ and is effectively incapable of revealing more than a few correct digits of $\mathcal{I}$ in practice.

In contrast, the TD method achieves exponential convergence in all cases, obtaining 12 or more correct digits with as few as 25 cubature points per dimension. For example, in the common-tetrahedron case with $N=15$, the TD method requires a total of $N^{2}=225$ integrand samples to achieve 10digit accuracy, while the SIR method requires $15^{4}=50,625$ samples to achieve roughly 2-digit accuracy.

\section{CONClusions}

In this paper I extended the Taylor-Duffy approach to singular Galerkin integrals, which had previously been ap- 
plied to integrals over triangle-product domains [8], [9] to the more challenging case of tetrahedron-product domains. As I demonstrated, this yields an algorithm for computing VIE matrix elements with accuracy and efficiency exceeding those of existing methods by several orders of magnitude. I am hopeful that this new technique and its free-software implementation [25] will prove useful for accelerating VIE solvers for electromagnetic scattering and other physical applications. Meanwhile, the successful extension from triangles to tetrahedra testifies to the broad generality of the basic TaylorDuffy strategy - and suggests that the full extent of its utility remains far from fully explored.

\begin{tabular}{|c|c|c|c|c|c|c|}
\hline$d$ & $u_{1 d}^{\mathrm{MIN}}$ & $u_{1 d}^{\mathrm{MAX}}$ & $u_{2 d}^{\mathrm{MIN}}$ & $u_{2 d}^{\mathrm{MAX}}$ & $u_{3 d}^{\mathrm{MIN}}$ & $u_{3 d}^{\mathrm{MAX}}$ \\
\hline 1 & 0 & 1 & $u_{1}$ & 1 & $u_{2}$ & 1 \\
\hline 2 & 0 & 1 & $u_{1}$ & 1 & 0 & $u_{2}$ \\
\hline 3 & 0 & 1 & $u_{1}$ & 1 & $u_{2}-1$ & 0 \\
\hline 4 & 0 & 1 & 0 & $u_{1}$ & $u_{2}$ & $1-u_{1}+u_{2}$ \\
\hline 5 & 0 & 1 & 0 & $u_{1}$ & 0 & $u_{2}$ \\
\hline 6 & 0 & 1 & 0 & $u_{1}$ & $u_{1}-1$ & 0 \\
\hline 7 & 0 & 1 & $u_{1}-1$ & 0 & 0 & $1-u_{1}+u_{2}$ \\
\hline 8 & 0 & 1 & $u_{1}-1$ & 0 & $u_{2}$ & 0 \\
\hline 9 & 0 & 1 & $u_{1}-1$ & 0 & $u_{1}-1$ & $u_{2}$ \\
\hline 10 & -1 & 0 & 0 & $u_{1}+1$ & $u_{2}$ & $1+u_{1}$ \\
\hline 11 & -1 & 0 & 0 & $u_{1}+1$ & 0 & $u_{2}$ \\
\hline 12 & -1 & 0 & 0 & $u_{1}+1$ & $u_{2}-u_{1}-1$ & 0 \\
\hline 13 & -1 & 0 & $u_{1}$ & 0 & 0 & $u_{1}+1$ \\
\hline 14 & -1 & 0 & $u_{1}$ & 0 & $u_{2}$ & 0 \\
\hline 15 & -1 & 0 & $u_{1}$ & 0 & $u_{2}-u_{1}-1$ & $u_{2}$ \\
\hline 16 & -1 & 0 & -1 & $u_{1}$ & 0 & $1+u_{2}$ \\
\hline 17 & -1 & 0 & -1 & $u_{1}$ & $u_{2}$ & 0 \\
\hline 18 & -1 & 0 & -1 & $u_{1}$ & -1 & $u_{2}$ \\
\hline
\end{tabular}

TABLE III

LIMITS OF INTEGRATION FOR THE TETRAHEDRAL DOMAINS $\triangle_{d}^{\mathbf{u}}$ IN DEFINED BY $\int_{\triangle_{d}} d \mathbf{u} \equiv \int_{u_{1 d}^{\mathrm{MIN}}}^{u_{1 d}^{\mathrm{MAX}}} d u_{1} \int_{u_{2 d}^{\mathrm{MIN}}}^{u_{2 d}^{\mathrm{MAX}}} d u_{2} \int_{u_{3 d}^{\mathrm{MIN}}}^{u_{3 d}^{\mathrm{MAX}}} d u_{3}$.

\begin{tabular}{|c|c|c|c|c|c|c|}
\hline$d$ & $L_{3 d}$ & $U_{3 d}$ & $L_{2 d}$ & $U_{2 d}$ & $L_{1 d}$ & $U_{1 d}$ \\
\hline 1 & 0 & $-u_{3}$ & $u_{3}-u_{2}$ & $-u_{2}$ & $u_{2}-u_{1}$ & $-u_{1}$ \\
\hline 2 & 0 & $-u_{2}$ & 0 & $-u_{2}$ & $u_{2}-u_{1}$ & $-u_{1}$ \\
\hline 3 & $-u_{3}$ & $-u_{2}$ & 0 & $-u_{2}$ & $u_{2}-u_{1}$ & $-u_{1}$ \\
\hline 4 & 0 & $u_{2}-u_{1}-u_{3}$ & $u_{3}-u_{2}$ & $-u_{1}$ & 0 & $-u_{1}$ \\
\hline 5 & 0 & $-u_{1}$ & 0 & $-u_{1}$ & 0 & $-u_{1}$ \\
\hline 6 & $-u_{3}$ & $-u_{1}$ & 0 & $-u_{1}$ & 0 & $-u_{1}$ \\
\hline 7 & 0 & $u_{2}-u_{1}-u_{3}$ & $u_{3}-u_{2}$ & $-u_{1}$ & 0 & $-u_{1}$ \\
\hline 8 & $-u_{3}$ & $u_{2}-u_{1}-u_{3}$ & $u_{3}-u_{2}$ & $-u_{1}$ & 0 & $-u_{1}$ \\
\hline 9 & $-u_{3}$ & $-u_{1}$ & 0 & $-u_{1}$ & 0 & $-u_{1}$ \\
\hline 10 & 0 & $u_{1}-u_{3}$ & $u_{3}-u_{2}$ & $u_{1}-u_{2}$ & $u_{2}-u_{1}$ & 0 \\
\hline 11 & 0 & $u_{1}-u_{2}$ & 0 & $u_{1}-u_{2}$ & $u_{2}-u_{1}$ & 0 \\
\hline 12 & $-u_{3}$ & $u_{1}-u_{2}$ & 0 & $u_{1}-u_{2}$ & $u_{2}-u_{1}$ & 0 \\
\hline 13 & 0 & $u_{1}-u_{3}$ & $u_{3}-u_{2}$ & $u_{1}-u_{2}$ & $u_{2}-u_{1}$ & 0 \\
\hline 14 & $-u_{3}$ & $u_{1}-u_{3}$ & $u_{3}-u_{2}$ & $u_{1}-u_{2}$ & $u_{2}-u_{1}$ & 0 \\
\hline 15 & $-u_{3}$ & $u_{1}-u_{2}$ & 0 & $u_{1}-u_{2}$ & $u_{2}-u_{1}$ & 0 \\
\hline 16 & 0 & $u_{2}-u_{3}$ & $u_{3}-u_{2}$ & 0 & 0 & 0 \\
\hline 17 & $-u_{3}$ & $u_{2}-u_{3}$ & $u_{3}-u_{2}$ & 0 & 0 & 0 \\
\hline 18 & $-u_{3}$ & 0 & 0 & 0 & 0 & 0 \\
\hline
\end{tabular}

TABLE IV

LIMITS OF INTEGRATION FOR THE TETRAHEDRAL DOMAINS $\triangle_{d}^{\boldsymbol{\xi}}$ IN 4 , DEFINED BY $\int_{\triangle_{d}} d \boldsymbol{\xi} \equiv \int_{L_{3 d}}^{1+U_{3 d}} d \xi_{3} \int_{\xi_{3}+L_{2 d}}^{1+U_{2 d}} d \xi_{2} \int_{\xi_{2}+L_{1 d}}^{1+U_{1 d}} d \xi_{1}$.

\section{APPENDIX A}

TABles of Subdomain-DePEndent Quantities

Tables III VII provide explicit definitions of various subregion-dependent quantities referenced in Section III

\section{REFERENCES}

[1] R. F. Harrington, Field Computation by Moment Methods. Wiley-IEEE Press, 1993.

[2] W. Chew, M. Tong, and B. Hu, Integral Equation Methods for Electromagnetic and Elastic Waves, ser. Synthesis Lectures on Computational Electromagnetics Series. Morgan \& Claypool Publishers, 2009. [Online]. Available: http://books.google.com/books? id=PJN9meadzT8C

[3] S. K. Volakis, John. SciTech Publishing, 2012. [Online]. Available: http://app.knovel.com/hotlink/toc/id:kpIEME0011/ integral-equation-methods/integral-equation-methods 


\begin{tabular}{|c|c|c|c|c|}
\hline$d$ & $\mathcal{J}_{d}(\mathbf{y})$ & $u_{1}(w, \mathbf{y})$ & $u_{2}(w, \mathbf{y})$ & $u_{3}(w, \mathbf{y})$ \\
\hline 1 & $y_{1}$ & $w y_{1} y_{2}$ & $w y_{1}$ & $w$ \\
\hline 2 & 1 & $w y_{1}$ & $w$ & $w y_{2}$ \\
\hline 3 & $y_{1}$ & $w y_{1} y_{2}$ & $w y_{1}$ & $-w\left(1-y_{1}\right)$ \\
\hline 4 & $y_{1}$ & $w y_{1}$ & $w y_{1} y_{2}$ & $w\left(1-y_{1}+y_{1} y_{2}\right)$ \\
\hline 5 & $y_{1}$ & $w$ & $w y_{1}$ & $w y_{1} y_{2}$ \\
\hline 6 & $y_{1}$ & $w y_{1}$ & $w y_{1} y_{2}$ & $-w\left(1-y_{1}\right)$ \\
\hline 7 & $y_{1}$ & $w y_{1} y_{2}$ & $-w y_{1}\left(1-y_{2}\right)$ & $w\left(1-y_{1}\right)$ \\
\hline 8 & $y_{1}$ & $w\left(1-y_{1}\right)$ & $-w y_{1}$ & $-w y_{1} y_{2}$ \\
\hline 9 & $y_{1}$ & $w y_{1}\left(1-y_{2}\right)$ & $-w y_{1} y_{2}$ & $-w\left(1-y_{1}+y_{1} y_{2}\right)$ \\
\hline 10 & $y_{1}$ & $-w y_{1} y_{2}$ & $w y_{1}\left(1-y_{2}\right)$ & $w\left(1-y_{1} y_{2}\right)$ \\
\hline 11 & $y_{1}$ & $-w y_{1} y_{2}$ & $w\left(1-y_{1} y_{2}\right)$ & $w y_{1}\left(1-y_{2}\right)$ \\
\hline 12 & $y_{1}$ & $-w y_{1} y_{2}$ & $w y_{1}\left(1-y_{2}\right)$ & $-w\left(1-y_{1}\right)$ \\
\hline 13 & $y_{1}$ & $-w y_{1}$ & $-w y_{1} y_{2}$ & $w\left(1-y_{1}\right)$ \\
\hline 14 & $y_{1}$ & $-w$ & $-w y_{1}$ & $-w y_{1} y_{2}$ \\
\hline 15 & $y_{1}$ & $-w y_{1}$ & $-w y_{1} y_{2}$ & $-w\left(1-y_{1}+y_{1} y_{2}\right)$ \\
\hline 16 & $y_{1}$ & $-w y_{1} y_{2}$ & $-w y_{1}$ & $w\left(1-y_{1}\right)$ \\
\hline 17 & 1 & $-w y_{1}$ & $-w$ & $-w\left(1-y_{2}\right)$ \\
\hline 18 & $y_{1}$ & $-w y_{1} y_{2}$ & $-w y_{1}$ & $-w$ \\
\hline
\end{tabular}

TABLE V

DUFFY TRANSFORMATIONS FOR THE CASE $N_{\mathrm{CV}}=4$.

[4] E. Bleszynski, M. Bleszynski, and T. Jaroszewicz, "Fast volumetric integral-equation solver for acoustic wave propagation through inhomogeneous media," The Journal of the Acoustical Society of America, vol. 124, no. 1, 2008.

[5] J. Markkanen and P. Yl-Oijala, "Discretization of electric current volume integral equation with piecewise linear basis functions," IEEE Transactions on Antennas and Propagation, vol. 62, no. 9, pp. 4877-4880, Sept 2014.

[6] D. Schaubert, D. Wilton, and A. Glisson, "A tetrahedral modeling method for electromagnetic scattering by arbitrarily shaped inhomogeneous dielectric bodies," IEEE Transactions on Antennas and Propagation, vol. 32, no. 1, pp. 77-85, Jan 1984.

[7] M. G. Duffy, "Quadrature over a pyramid or cube of integrands with a singularity at a vertex," SIAM Journal on Numerical Analysis, vol. 19, no. 6, pp. 1260-1262, 1982.

[8] D. Taylor, "Accurate and efficient numerical integration of weakly singular integrals in Galerkin EFIE solutions," Antennas and Propagation, IEEE Transactions on, vol. 51, no. 7, pp. 1630-1637, 2003.

[9] M. Reid, J. White, and S. Johnson, "Generalized Taylor-Duffy method for efficient evaluation of galerkin integrals in boundary-element method computations," Antennas and Propagation, IEEE Transactions on, vol. 63, no. 1, pp. 195-209, Jan 2015.

[10] E. Bleszynski, M. Bleszynski, and T. Jaroszewicz, "Reduction of volume integrals to nonsingular surface integrals for matrix elements of tensor and vector green functions of maxwell equations," Antennas and Propagation, IEEE Transactions on, vol. 61, no. 7, pp. 3642-3647, July 2013.

[11] M. Hasanovic, C. Mei, J. R. Mautz, and E. Arvas, "Scattering from 3-d inhomogeneous chiral bodies of arbitrary shape by the method of moments," IEEE Transactions on Antennas and Propagation, vol. 55, no. 6, pp. 1817-1825, June 2007.

\begin{tabular}{|c|c|c|c|c|c|}
\hline$d$ & $\mathcal{J}_{d}(\mathbf{y})$ & $u_{1}(w, \mathbf{y})$ & $u_{2}(w, \mathbf{y})$ & $u_{3}(w, \mathbf{y})$ & $\xi_{3}(w, \mathbf{y})$ \\
\hline 1 & $y_{1}^{2} y_{2}$ & $w y_{1} y_{2} y_{3}$ & $w y_{1} y_{2}$ & $w y_{1}$ & $w \bar{y}_{1}$ \\
\hline 2 & $y_{1}^{2}$ & $w y_{1} y_{2}$ & $w y_{1}$ & $w y_{1} y_{3}$ & $w \bar{y}_{1}$ \\
\hline 3 & $y_{1}^{2} y_{2}$ & $w y_{1} y_{2} y_{3}$ & $w y_{1} y_{2}$ & $-w y_{1} \bar{y}_{2}$ & $w \zeta_{\mathrm{A}}$ \\
\hline 4 & $y_{1}^{2} y_{2}$ & $w y_{1} y_{2}$ & $w y_{1} y_{2} y_{3}$ & $w y_{1} \zeta_{\mathrm{D}}$ & $w \bar{y}_{1}$ \\
\hline 5 & $y_{1}^{2} y_{2}$ & $w y_{1}$ & $w y_{1} y_{2}$ & $w y_{1} y_{2} y_{3}$ & $w \bar{y}_{1}$ \\
\hline 6 & $y_{1}^{2} y_{2}$ & $w y_{1} y_{2}$ & $w y_{1} y_{2} y_{3}$ & $-w y_{1} \bar{y}_{2}$ & $w \zeta_{\mathrm{A}}$ \\
\hline 7 & $y_{1}^{2} y_{2}$ & $w y_{1} y_{2} y_{3}$ & $-w y_{1} y_{2} \bar{y}_{3}$ & $w y_{1} \bar{y}_{2}$ & $w \bar{y}_{1}$ \\
\hline 8 & $y_{1}^{2} y_{2}$ & $w y_{1} \bar{y}_{2}$ & $-w y_{1} y_{2}$ & $-w y_{1} y_{2} y_{3}$ & $w \zeta_{\mathrm{E}}$ \\
\hline 9 & $y_{1}^{2} y_{2}$ & $w y_{1} y_{2} \bar{y}_{3}$ & $-w y_{1} y_{2} y_{3}$ & $-w y_{1} \zeta_{\mathrm{D}}$ & $w \zeta_{\mathrm{F}}$ \\
\hline 10 & $y_{1}^{2} y_{2}$ & $-w y_{1} y_{2} y_{3}$ & $w y_{1} y_{2} \bar{y}_{3}$ & $w y_{1} \zeta_{\mathrm{C}}$ & $w \bar{y}_{1}$ \\
\hline 11 & $y_{1}^{2} y_{2}$ & $-w y_{1} y_{2} y_{3}$ & $w y_{1} \zeta_{\mathrm{C}}$ & $w y_{1} y_{2} \bar{y}_{3}$ & $w \bar{y}_{1}$ \\
\hline 12 & $y_{1}^{2} y_{2}$ & $-w y_{1} y_{2} y_{3}$ & $w y_{1} y_{2} \bar{y}_{3}$ & $-w y_{1} \bar{y}_{2}$ & $w \zeta_{\mathrm{A}}$ \\
\hline 13 & $y_{1}^{2} y_{2}$ & $-w y_{1} y_{2}$ & $-w y_{1} y_{2} y_{3}$ & $w y_{1} \bar{y}_{2}$ & $w \bar{y}_{1}$ \\
\hline 14 & $y_{1}^{2} y_{2}$ & $-w y_{1}$ & $-w y_{1} y_{2}$ & $-w y_{1} y_{2} y_{3}$ & $w \zeta_{\mathrm{D}}$ \\
\hline 15 & $y_{1}^{2} y_{2}$ & $-w y_{1} y_{2}$ & $-w y_{1} y_{2} y_{3}$ & $-w y_{1} \zeta_{\mathrm{D}}$ & $w \zeta_{\mathrm{F}}$ \\
\hline 16 & $y_{1}^{2} y_{2}$ & $-w y_{1} y_{2} y_{3}$ & $-w y_{1} y_{2}$ & $w y_{1} \bar{y}_{2}$ & $w \bar{y}_{1}$ \\
\hline 17 & $y_{1}^{2}$ & $-w y_{1} y_{2}$ & $-w y_{1}$ & $-w y_{1} \bar{y}_{3}$ & $w \zeta_{\mathrm{B}}$ \\
\hline 18 & $y_{1}^{2} y_{2}$ & $-w y_{1} y_{2} y_{3}$ & $-w y_{1} y_{2}$ & $-w y_{1}$ & $w$ \\
\hline
\end{tabular}

TABLE VI

DUFFY TRANSFORMATIONS FOR THE CASE $N_{\mathrm{CV}}=3$. SHORTHAND:

$\overline{y_{i}} \equiv 1-y_{i}, \zeta_{\mathrm{A}} \equiv 1-y_{1} y_{2}, \zeta_{\mathrm{B}} \equiv 1-y_{1} y_{3}, \zeta_{\mathrm{C}} \equiv 1-y_{2} y_{3}$, $\zeta_{\mathrm{D}} \equiv 1-y_{2}+y_{2} y_{3}, \zeta_{\mathrm{E}} \equiv 1-y_{1}+y_{1} y_{2} y_{3}, \zeta_{\mathrm{F}} \equiv 1-y_{1} y_{2}+y_{1} y_{2} y_{3}$.

[12] L. M. Zhang and X. Q. Sheng, "Solving volume electric current integral equation with full- and half-SWG functions," IEEE Antennas and Wireless Propagation Letters, vol. 14, pp. 682-685, 2015.

[13] K. Jackman and C. Fourie, "Tetrahedral modeling method for inductance extraction of complex 3-d superconducting structures," IEEE Transactions on Applied Superconductivity, vol. 26, no. 3, 2016, cited By 0. [Online]. Available: https://www.scopus.com/inward/record.uri?eid=2-s2.0-84963795087\& partnerID=40\&md5=0c1d22abfe016803c1242966982aef81

[14] M. T. H. Reid, "Efficient Computation of Power, Force and Torque in Integral-Equation Solvers: Nonsingular Integrals and Moment Expansions", to appear.

[15] M. M. Botha, "Solving the volume integral equations of electromagnetic scattering," Journal of Computational Physics, vol. 218, no. 1, pp. 141 - 158, 2006. [Online]. Available: http://www.sciencedirect.com/science/ article/pii/S0021999106000763

[16] J. Markkanen, C. C. Lu, X. Cao, and P. Yla-Oijala, "Analysis of volume integral equation formulations for scattering by high-contrast penetrable objects," IEEE Transactions on Antennas and Propagation, vol. 60, no. 5, pp. 2367-2374, May 2012.

[17] A. Polimeridis, J. Villena, L. Daniel, and J. White, "Stable FFTJVIE solvers for fast analysis of highly inhomogeneous dielectric objects," Journal of Computational Physics, vol. 269, pp. $280-296$, 2014. [Online]. Available: http://www.sciencedirect.com/science/article/ pii/S0021999114002071

[18] R. Cools, "An encyclopaedia of cubature formulas," Journal of Complexity, vol. 19, no. 3, pp. 445 - 453, 2003, oberwolfach Special Issue. [Online]. Available: http://www.sciencedirect.com/science/article/ pii/S0885064X03000116 


\begin{tabular}{|c|c|c|c|c|c|c|}
\hline$d$ & $\mathcal{J}_{d}$ & $\frac{u_{1}}{w}$ & $\frac{u_{2}}{w}$ & $\frac{u_{3}}{w}$ & $\frac{\xi_{3}}{w}$ & $\frac{\xi_{2}}{w}$ \\
\hline 1 & $y_{1}^{3} y_{2}^{2} y_{3}$ & $y_{1} y_{2} y_{3} y_{4}$ & $y_{1} y_{2} y_{3}$ & $y_{1} y_{2}$ & $y_{1} \bar{y}_{2}$ & $\Upsilon_{\mathrm{E}}$ \\
\hline 2 & $y_{1}^{3} y_{2}^{2}$ & $y_{1} y_{2} y_{4}$ & $y_{1} y_{2}$ & $y_{1} y_{2} y_{3}$ & $y_{1} \bar{y}_{2}$ & $\Upsilon_{\mathrm{A}}$ \\
\hline 3 & $y_{1}^{3} y_{2}^{2} y_{3}$ & $y_{1} y_{2} y_{3} y_{4}$ & $y_{1} y_{2} y_{3}$ & $-y_{1} y_{2} \bar{y}_{3}$ & $y_{1} \Upsilon_{\mathrm{B}}$ & $\Upsilon_{\mathrm{E}}$ \\
\hline 4 & $y_{1}^{3} y_{2}^{2} y_{3}$ & $y_{1} y_{2} y_{3}$ & $y_{1} y_{2} y_{3} y_{4}$ & $y_{1} y_{2} \Upsilon_{\mathrm{G}}$ & $y_{1} \bar{y}_{2}$ & $\Upsilon_{\mathrm{E}}$ \\
\hline 5 & $y_{1}^{3} y_{2}^{2} y_{3}$ & $y_{1} y_{2}$ & $y_{1} y_{2} y_{3}$ & $y_{1} y_{2} y_{3} y_{4}$ & $y_{1} \bar{y}_{2}$ & $\Upsilon_{\mathrm{A}}$ \\
\hline 6 & $y_{1}^{3} y_{2}^{2} y_{3}$ & $y_{1} y_{2} y_{3}$ & $y_{1} y_{2} y_{3} y_{4}$ & $-y_{1} y_{2} \overline{y_{3}}$ & $y_{1} \Upsilon_{\mathrm{B}}$ & $\Upsilon_{\mathrm{E}}$ \\
\hline 7 & $y_{1}^{3} y_{2}^{2} y_{3}$ & $y_{1} y_{2} y_{3} y_{4}$ & $-y_{1} y_{2} y_{3} \bar{y}_{4}$ & $y_{1} y_{2} \bar{y}_{3}$ & $y_{1} \bar{y}_{2}$ & $\Upsilon_{\mathrm{F}}$ \\
\hline 8 & $y_{1}^{3} y_{2}^{2} y_{3}$ & $y_{1} y_{2} \bar{y}_{3}$ & $-y_{1} y_{2} y_{3}$ & $-y_{1} y_{2} y_{3} y_{4}$ & $y_{1} \Upsilon_{\mathrm{H}}$ & $\Upsilon_{I}$ \\
\hline 9 & $y_{1}^{3} y_{2}^{2} y_{3}$ & $y_{1} y_{2} y_{3} \bar{y}_{4}$ & $-y_{1} y_{2} y_{3} y_{4}$ & $-y_{1} y_{2} \Upsilon_{\mathrm{G}}$ & $y_{1} \Upsilon_{\mathrm{J}}$ & $\Upsilon_{\mathrm{K}}$ \\
\hline 10 & $y_{1}^{3} y_{2}^{2} y_{3}$ & $-y_{1} y_{2} y_{3} y_{4}$ & $y_{1} y_{2} y_{3} \bar{y}_{4}$ & $y_{1} y_{2} \Upsilon_{\mathrm{D}}$ & $y_{1} \bar{y}_{2}$ & $\Upsilon_{E}$ \\
\hline 11 & $y_{1}^{3} y_{2}^{2} y_{3}$ & $-y_{1} y_{2} y_{3} y_{4}$ & $y_{1} y_{2} \Upsilon_{\mathrm{D}}$ & $y_{1} y_{2} y_{3} \bar{y}_{4}$ & $y_{1} \bar{y}_{2}$ & $\Upsilon_{\mathrm{A}}$ \\
\hline 12 & $y_{1}^{3} y_{2}^{2} y_{3}$ & $-y_{1} y_{2} y_{3} y_{4}$ & $y_{1} y_{2} y_{3} \bar{y}_{4}$ & $-y_{1} y_{2} \bar{y}_{3}$ & $y_{1} \Upsilon_{\mathrm{B}}$ & $\Upsilon_{\mathrm{E}}$ \\
\hline 13 & $y_{1}^{3} y_{2}^{2} y_{3}$ & $-y_{1} y_{2} y_{3}$ & $-y_{1} y_{2} y_{3} y_{4}$ & $y_{1} y_{2} \bar{y}_{3}$ & $y_{1} \bar{y}_{2}$ & $\Upsilon_{K}$ \\
\hline 14 & $y_{1}^{3} y_{2}^{2} y_{3}$ & $-y_{1} y_{2}$ & $-y_{1} y_{2} y_{3}$ & $-y_{1} y_{2} y_{3} y_{4}$ & $y_{1} \Upsilon_{\mathrm{H}}$ & $\Upsilon_{\mathrm{I}}$ \\
\hline 15 & $y_{1}^{3} y_{2}^{2} y_{3}$ & $-y_{1} y_{2} y_{3}$ & $-y_{1} y_{2} y_{3} y_{4}$ & $-y_{1} y_{2} \Upsilon_{\mathrm{G}}$ & $y_{1} \Upsilon_{\mathrm{J}}$ & $\Upsilon_{K}$ \\
\hline 16 & $y_{1}^{3} y_{2}^{2} y_{3}$ & $-y_{1} y_{2} y_{3} y_{4}$ & $-y_{1} y_{2} y_{3}$ & $y_{1} y_{2} \bar{y}_{3}$ & $y_{1} \bar{y}_{2}$ & 1 \\
\hline 17 & $y_{1}^{3} y_{2}^{2} y_{3}$ & $-y_{1} y_{2} y_{3}$ & $-y_{1} y_{2}$ & $-y_{1} y_{2} \bar{y}_{4}$ & $y_{1} \Upsilon_{\mathrm{C}}$ & 1 \\
\hline 18 & $y_{1}^{3} y_{2}^{2} y_{3}$ & $-y_{1} y_{2} y_{3} y_{4}$ & $-y_{1} y_{2} y_{3}$ & $-y_{1} y_{2}$ & $y_{1}$ & 1 \\
\hline
\end{tabular}

TABLE VII

DUFFY TRANSFORMATIONS FOR THE CASE $N_{\mathrm{CV}}=2$. (NOTE THAT A FACTOR OF $w$ HAS BEEN EXTRACTED FROM EACH $\mathbf{u}, \boldsymbol{\xi}$ COMPONENT TO SAVE SPACE). SHORTHAND: $\bar{y}_{i} \equiv 1-y_{i}, \Upsilon_{\mathrm{A}} \equiv 1-y_{1} y_{2}$,

$\Upsilon_{\mathrm{B}} \equiv 1-y_{2} y_{3}, \Upsilon_{\mathrm{C}} \equiv 1-y_{2} y_{4}, \Upsilon_{\mathrm{D}} \equiv 1-y_{3} y_{4}, \Upsilon_{\mathrm{E}} \equiv 1-y_{1} y_{2} y_{3}$, $\Upsilon_{\mathrm{F}} \equiv 1-y_{1} y_{2} y_{3} y_{4}, \Upsilon_{\mathrm{G}} \equiv 1-y_{3}+y_{3} y_{4}, \Upsilon_{\mathrm{H}} \equiv 1-y_{2}+y_{2} y_{3} y_{4}$, $\Upsilon_{\mathrm{I}} \equiv 1-y_{1} y_{2}+y_{1} y_{2} y_{3}, \Upsilon_{\mathrm{J}} \equiv 1-y_{2} y_{3}+y_{2} y_{3} y_{4}$,

$$
\Upsilon_{\mathrm{K}} \equiv 1-y_{1} y_{2} y_{3}+y_{1} y_{2} y_{3} y_{4} \text {. }
$$

[19] S. Rao, D. Wilton, and A. Glisson, "Electromagnetic scattering by surfaces of arbitrary shape," Antennas and Propagation, IEEE Transactions on, vol. 30, no. 3, pp. 409-418, May 1982.

[20] L. N. Medgyesi-Mitschang, J. M. Putnam, and M. B. Gedera, "Generalized method of moments for three-dimensional penetrable scatterers," J. Opt. Soc. Am. A, vol. 11, no. 4, pp. 1383-1398, Apr 1994. [Online]. Available: http://josaa.osa.org/abstract.cfm?URI= josaa-11-4-1383

[21] L. Hu, L.-W. Li, T.-S. Yeo, and R. Vahldieck, "An accurate and robust approach for evaluating vie impedance matrix elements using SWG basis functions," in Microwave Conference, 2008. APMC 2008. Asia-Pacific, Dec 2008, pp. 1-4.

[22] S. Jrvenp, M. Taskinen, and P. Y1-Oijala, "Singularity extraction technique for integral equation methods with higher order basis functions on plane triangles and tetrahedra," International Journal for Numerical Methods in Engineering, vol. 58, no. 8, pp. 1149-1165, 2003. [Online]. Available: http://dx.doi.org/10.1002/nme.810

[23] D. Wilton, S. Rao, A. Glisson, D. Schaubert, O. Al-Bundak, and C. Butler, "Potential integrals for uniform and linear source distributions on polygonal and polyhedral domains," IEEE Transactions on Antennas and Propagation, vol. 32, no. 3, pp. 276-281, Mar 1984.

[24] R. Burns, B. Dubrovin, A. Fomenko, and S. Novikov, Modern Geometry Methods and Applications: Part II: The Geometry and Topology of Manifolds, ser. Graduate Texts in Mathematics. Springer New York, 1985. [Online]. Available: https://books.google.com/books? id=tlzc7xXYKd8C

[25] https://github.com/HomerReid/buff-em.

[26] J. Markkanen and P. Y1-Oijala, "Discretization of electric current volume integral equation with piecewise linear basis functions," IEEE Transac- tions on Antennas and Propagation, vol. 62, no. 9, pp. 4877-4880, Sept 2014.

[27] J. Markkanen, C. C. Lu, X. Cao, and P. Yla-Oijala, "Analysis of volume integral equation formulations for scattering by high-contrast penetrable objects," IEEE Transactions on Antennas and Propagation, vol. 60, no. 5, pp. 2367-2374, May 2012.

[28] L. Trefethen, Spectral Methods in MATLAB, ser. Software, Environments, and Tools. Society for Industrial and Applied Mathematics, 2000. [Online]. Available: https://books.google.cz/books? $\mathrm{id}=\mathrm{pB} 4 \mathrm{xiZKZ4ecC}$ 